\title{
The Dissolution of Double Holliday Junctions
}

\author{
Anna H. Bizard and lan D. Hickson \\ Nordea Center for Healthy Aging, Department of Cellular and Molecular Medicine, Panum Institute, \\ University of Copenhagen, 2200 Copenhagen N, Denmark \\ Correspondence: iandh@sund.ku.dk
}

Double Holliday junctions $(\mathrm{dHJS})$ are important intermediates of homologous recombination. The separate junctions can each be cleaved by DNA structure-selective endonucleases known as Holliday junction resolvases. Alternatively, double Holliday junctions can be processed by a reaction known as "double Holliday junction dissolution." This reaction requires the cooperative action of a so-called "dissolvasome" comprising a Holliday junction branch migration enzyme (Sgs1/BLM RecQ helicase) and a type IA topoisomerase (Top3/ Topolll $\alpha$ ) in complex with its OB (oligonucleotide/oligosaccharide binding) fold containing accessory factor (Rmi1). This review details our current knowledge of the dissolution process and the players involved in catalyzing this mechanistically complex means of completing homologous recombination reactions.

For decades, homologous recombination F (HR) was defined as a mechanism for the production of new allelic combinations during meiosis because it can generate so-called crossing-over (see Mehta and Haber 2014). Crossovers are likely generated by the asymmetric cleavage of a key intermediate in $\mathrm{HR}$, the $\mathrm{dHJ}$, by the action of structure-selective endonucleases called "resolvases" (Fig. 1A) (see Wyatt and West 2014). In addition to its essential function during meiosis, HR has proven to be a crucial DNA repair pathway in mitotic cells. Precisely because it has the potential to generate crossingover, the resolution of $\mathrm{dHJ}$ by resolvases affords a high risk of genomic instability in these circumstances. Indeed, when HR is engaged between two homologous chromosomes or two homeologous sequences, $\mathrm{dHJ}$ resolution could lead, respectively, to loss of heterozygosity or gross chromosomal rearrangements. Thus, an alternative mechanism allowing $\mathrm{dHJ}$ processing without crossing-over would appear essential when HR is used for DNA repair. Such a mechanism, termed $\mathrm{dHJ}$ dissolution, is thought to be a major route for dissipation of $\mathrm{dHJs}$ arising from HR repair (LaRocque et al. 2011; Krejci et al. 2012). During dHJ dissolution, the two HJs are branch migrated toward one another until they form a hemicatenated intermediate that can be decatenated by a topoisomerase (Fig. 1B). This sophisticated reaction is performed by the so-called "dissolvasome" complex composed of a specific RecQ helicase (BLM in humans/Sgs1 in budding yeast) and a type IA topoisomerase known as topoisomerase III (Fig. 2; for general reviews about RecQ helicases and topoisomerases, see Champoux 2001; Wang 2002; Bachrati and Hickson 2003; Viard and de la Tour 2007; Chu and Hickson 2009; Vindigni and Hickson 2009.

Editors: Stephen Kowalczykowski, Neil Hunter, and Wolf-Dietrich Heyer

Additional Perspectives on DNA Recombination available at www.cshperspectives.org

Copyright (C) 2014 Cold Spring Harbor Laboratory Press; all rights reserved; doi: 101101/cshperspect.a016477

Cite this article as Cold Spring Harb Perspect Biol 2014;6:a016477 
A.H. Bizard and I.D. Hickson

\section{A}

Resolution

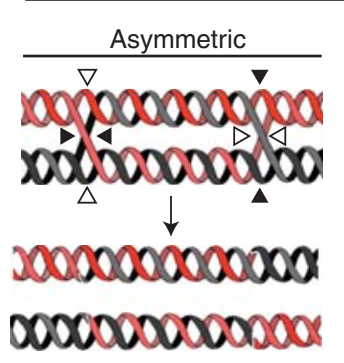

Crossover



Noncrossover

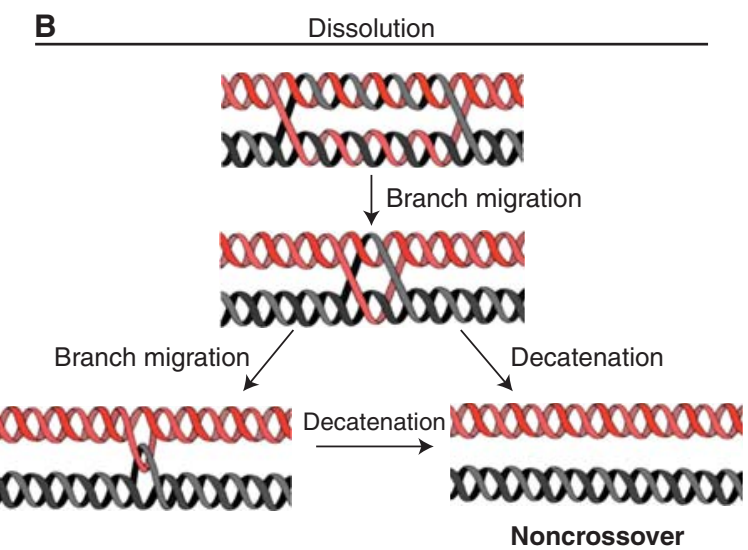

Figure 1. Double Holliday junction processing pathways. (A) During HJ resolution, each HJ of a dHJ is cleaved by a structure-selective endonuclease (resolvase). Depending on the combination of cleavage orientations, which can be asymmetric or symmetric, this process can generate both crossover and noncrossover products. In contrast, during dissolution $(B)$, each strand engaged in the $\mathrm{dHJ}$ is reassociated with its original complementary strand, preventing exchange of genetic material between the two homologous sequences (and hence generating exclusively noncrossover products). DHJ dissolution $(B)$ is initiated by migration of the HJs toward one another. The fusion/collapse of the two HJs results in a hemicatenated intermediate. Decatenation of this intermediate regenerates the original DNA species present before the initiation of HR.

In this review, we first take a historical look at the experimental evidence that led some groups to formulate the proposal that a reaction akin to dissolution must exist, and which then led Wu and Hickson (2003) to confirm its existence by reconstitution of the dissolution reaction in vitro using purified proteins. Following that, we will review the individual and combined roles of the components of what we will term the $\mathrm{dHJ}$ dissolvasome. Although many mechanistic aspects of $\mathrm{dHJ}$ dissolution remain obscure, several biochemical studies have provided a general understanding of this conceptually simple, but mechanistically complex, reaction.

\section{BACKGROUND TO THE DISCOVERY OF DHJ DISSOLUTION}

Well before the discovery of the $\mathrm{dHJ}$ dissolution process, the two catalytic subunits of the dissolvasome, a specific RecQ helicase and a type IA topoisomerase, were individually known for their crucial role in preventing genomic instability generated during HR.

\section{Background on RecQ Helicases}

Although the first RecQ helicase family member to be identified was RecQ of Escherichia coli, this review will focus on the eukaryotic homologs, 
Dissolution of Double Holliday Junctions

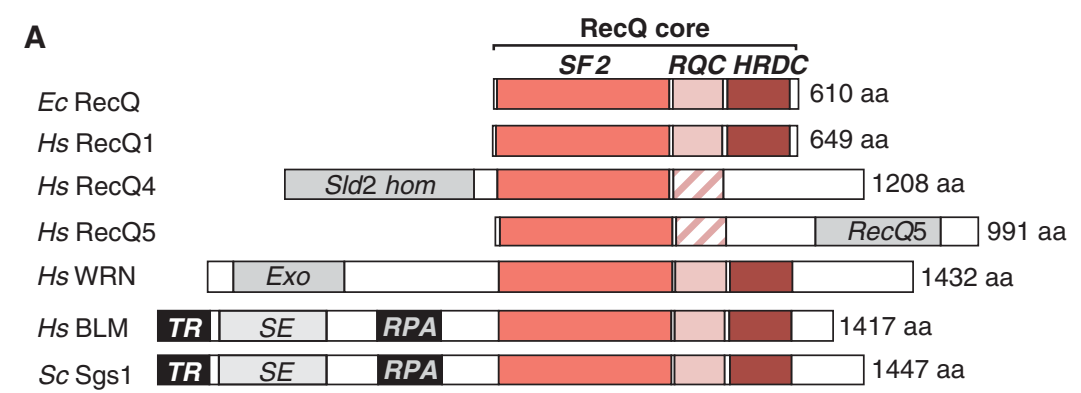

B

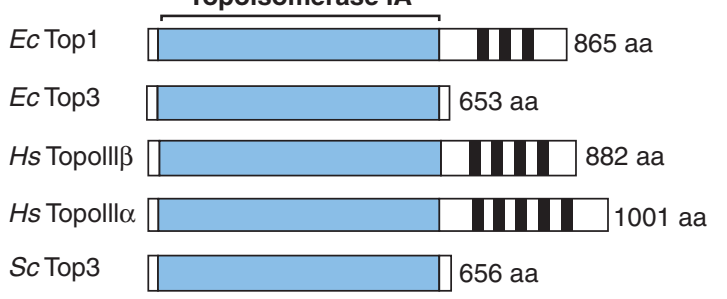

C

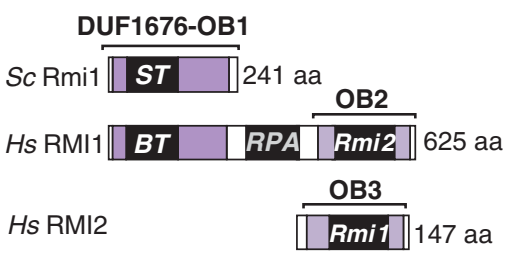

Figure 2. Domain organization of RecQ helicases, topoisomerases IA, and RMI proteins. (A) Most of the RecQ helicase members share a superfamily 2 helicase domain (SF2), a RecQ conserved domain (RQC), and a helicase and RNase D carboxy-terminal domain (HRDC). Besides this "RecQ core" domain, some RecQ helicases contain amino-terminal and carboxy-terminal extensions that vary in size, sequence, and functionality (e.g., SLD2 homology domain in RECQ4, and a signature motif in the carboxy-terminal domain of RECQ5). The hatched boxes denote partially degenerate RQC domains. BLM/Sgs1 helicases share a common domain organization, including an amino-terminal extension that includes domains for interaction with both TopoIII/ RMI1 (TR) and replication protein A (RPA), in addition to a region that has been proposed to be required for DNA strand exchange (SE) activity. (B) All type IA topoisomerases contain a conserved catalytic domain (topoisomerase IA). Some topoisomerase IA enzymes also exhibit a carboxy-terminal extension, frequently composed of zinc finger motifs (black boxes), which is believed to mediate protein-DNA and protein-protein interactions. The contribution of the carboxy-terminal extension to dissolution is unknown. The regions interacting with other components of the dissolvasome are unknown. $(C)$ In RMI1 proteins, only the DUF1676 and the OB-fold domain 1 (OB1) are conserved from yeast to human. The OB1 associates with both BLM/Sgs1 and topoisomerase III (BT/ST). In addition, human RMI1 exhibits a carboxy-terminal extension, composed of a middle region, which mediates RPA binding, and a second OB fold (OB2), which is able to associate with RMI2. RMI2 is also an OB-fold protein (OB3) that stably associates with the dissolvasome in human cells. In total, therefore, the human RMI1/2 complex contains three OB folds.

because they are better characterized in terms of their interactions with $\mathrm{dHJs}$ (for a detailed review on RecQ of E. coli, see Nakayama 2005). Nevertheless, it is clear that the malfunction of a RecQ helicase causes genome instability in all organisms (for review, see Chu and Hickson 2009; Bernstein et al. 2010; Larsen and Hickson 2013). In particular, a consistent observation in recQ helicase mutants is an increase in the frequency of crossing-over, which is generally detected by scoring the level of sister chromatid exchanges (SCEs) or loss of heterozygosity between homologs. For example, Saccharomyces cerevisiae sgs 1 mutants show elevated levels of mitotic HR, illegitimate recombination (Gangloff et al. 1994; Watt et al. 1996; Yamagata et al. 1998; Onoda et al. 2000), and gross chromosomal rearrangements (Myung et al. 2001; Myung and Kolodner 2002). More specifically, Ira et al. (2003) showed that sgs 1 mutants have an elevated frequency of crossing-over during HR, suggesting a role of Sgs1 in crossover sup- 
pression. Among the five human RecQ homologs, BLM is believed to be an Sgs1 ortholog. Sgs1 and BLM share similar sequences, domain architecture, and functionality (Fig. 2) (Kusano et al. 1999). BLM is defective in a rare autosomal recessive disorder called Bloom's syndrome (BS) characterized by growth retardation, immune deficiency, reduced fertility, sensitivity to sunlight, and a high risk of developing various types of cancer (German 1993; Luo et al. 2000). Cells derived from BS patients show genomic instability, including the hallmark feature of an elevated frequency of crossover recombinationas scored by measuring SCE (Chaganti et al. 1974; German 1993).

\section{Background on Type IA Topoisomerases}

All topoisomerases are crucial for the proper transmission of the genetic information caused by their essential role during maintenance of cellular DNA supercoiling homeostasis and decatenation of sister chromatids in mitosis (for review, see Champoux 2001; Wang 2002; Viard and de la Tour 2007). It is worth noting that a subclass of topoisomerases, known as the type IA topoisomerase, plays little or no direct role in chromosome segregation or in regulating DNA supercoiling. Nevertheless, type IA topoisomerases are strongly conserved throughout evolution (Fig. 2) (Forterre and Gadelle 2009), and their essential nature has been emphasized by a plethora of genetic studies. Higher eukaryotic cells have two type IA topoisomerases, TopoIII $\alpha$ and TopoIII $\beta$. Although TopoIII $\alpha$ is involved in $\mathrm{DHJ}$ dissolution, the function of TopoIII $\beta$ remains unclear and will not be discussed further here. Deletion of the gene encoding TopoIII $\alpha$ leads to a lethal phenotype associated with severe developmental defects in mice, Drosophila melanogaster, and Arabidopsis thaliana (Li and Wang 1998; Plank et al. 2005; Hartung et al. 2007). In chicken DT40 cells, TopoIII $\alpha$ depletion also causes cell death associated with dramatic genomic instability (Seki et al. 2006). In Schizosaccharomyces pombe, deletion of top $3^{+}$is also lethal and cell death is associated with chromosome missegregation (Goodwin et al. 1999; Maftahi et al. 1999; Oh et al. 2002). In contrast,
S. cerevisiae top $3 \Delta$ strains are viable, but are very slow growing, and show a pleiotropic phenotype, including hyperrecombination, sensitivity to DNA-damaging agents, defects in accurate chromosome segregation, and a failure to complete meiosis (Wallis et al. 1989; Gangloff et al. 1994; Chakraverty et al. 2001).

\section{Genetic and Physical Interactions between RecQ Helicases and Topoisomerase III}

A possible functional interaction between the Sgs1/BLM RecQ helicases and type IA topoisomerases was first suggested by studies showing that mutations in their genes genetically interact. For the most part, mutations in SGS1 and $T O P 3$ are epistatic, and indeed deletion of SGS1 is a strong suppressor of the drastic growth and DNA repair defects in top3 mutants (Gangloff et al. 1994, 1999; Watt et al. 1996). A similar genetic interaction has also been found in S. pombe, where deletion of the SGS1 homolog, $r q h 1^{+}$, can rescue the lethality associated with top3s (Goodwin et al. 1999; Maftahi et al. 1999). In DT40 cells, the disruption of the RecQ helicase BLM suppresses genomic instability defects associated with TopIII $\alpha$ depletion, without actually preventing cell death (Seki et al. 2006). In addition to this conserved genetic interaction, Sgs1/BLM RecQ helicases and type IA topoisomerases physically interact, as has been shown in S. cerevisiae between Top3 and Sgs1 (Bennett et al. 2000; Fricke et al. 2001), in S. pombe between Top3 and Rqh1 (Laursen et al. 2003; Ahmad and Stewart 2005), and in human cells between BLM and TopoIII $\alpha$ (Johnson et al. 2000; Wu et al. 2000). More important, this physical interaction is crucial for Sgs1/BLM to maintain genomic stability in vivo, because the first 100 aa residues of Sgs1, which mediate the physical association with Top3, are required for the complementation of the sgs1 phenotypes (Gangloff et al. 1994; Bennett et al. 2000; Duno et al. 2000; Mullen et al. 2000; Fricke et al. 2001; Ui et al. 2001; Onodera et al. 2002; Ira et al. 2003; Weinstein and Rothstein 2008). Similarly, the putative TopoIII $\alpha$ interaction domain of BLM is necessary for the suppression of the elevated SCE 
phenotype of BS cells (Wu et al. 2000; Hu et al. 2001).

Connections between RecQ Helicases/Type IA Topoisomerases and HR

The hyper-crossing-over phenotypes of cells lacking Sgs1/BLM and Top3/TopoIII $\alpha$ suggest a role in HR. The search for other (non-sgs1) suppressors of top $3 \Delta$ growth impairment in $S$. cerevisiae and $S$. pombe identified genes encoding proteins involved in the initial steps of $\mathrm{HR}$, such as RAD51 and $\mathrm{rad} 22^{+}$, respectively (Oakley et al. 2002; Shor et al. 2002; Laursen et al. 2003). Moreover, sgs $1 /$ top 3 mutants accumulate unprocessed HR DNA intermediates following exposure to a DNA damaging agent (Liberi et al. 2005; Mankouri and Hickson 2006; Carotenuto and Liberi 2010). These data indicated that RecQ helicase/topoisomerase III act on DNA intermediates that build up during HR. The identification of those intermediates as being HJs was suggested by three additional facts. First, the viability of cells lacking BLM, Sgs1, and/or Top3 depends on functional DNA structure-selective endonucleases implicated as $\mathrm{HJ}$ resolvases such as Mus81-Mms4 (Kaliraman et al. 2001; Mullen et al. 2001; Andersen et al. 2011; Wechsler et al. 2011). Second, the synthetic lethality of $s g s 1$ mus81 double mutants can be suppressed either by the overexpression of the bacterial HJ resolvase, RusA, or by the inactivation of the early steps of HR (Mullen et al. 2001; Fabre et al. 2002; Bastin-Shanower et al. 2003). Similar results were obtained in $S$. pombe (Boddy et al. 2000; Doe et al. 2002), and in D. melanogaster (Trowbridge et al. 2007; Andersen et al. 2011). Third, it was demonstrated that the HR intermediates accumulating in $s g s 1$ and top3 mutants during a perturbed $S$-phase contain HJs (Bzymek et al. 2010; Ashton et al. 2011; Mankouri et al. 2011).

These genetic studies indicate that the RecQ-topoisomerase III complex actually plays a role in completion of $\mathrm{HR}$, representing an alternative to the classical $\mathrm{HJ}$ resolvase pathway for the dissipation of $\mathrm{dHJ}$ in a way that prevents crossing-overs. Several groups proposed that two HJs could theoretically be dissipated with- out resolvases by a two-step mechanism involving the convergent branch migration of $\mathrm{dHJ}$, followed by the release of hemicatenanes generated after the collapse of the two junctions (Fig. 1B) (Nasmyth 1982; Wang et al. 1990; Duguet 1997; Wu and Hickson 2001; Ira et al. 2003). Using an oligo-based dHJ substrate (OligoDHJ; Fig. 3), Wu and Hickson (2003) demonstrated that the BLM/TopoIII $\alpha$ complex catalyzes such a reaction in vitro, shedding light on most of the observations accumulated during years of genetic analysis on those enzymes.

\section{MECHANISTIC INSIGHT INTO THE DISSOLUTION OF A DOUBLE $\mathrm{HJ}(\mathrm{dHJ})$}

Two substrates are commonly used to study dissolution in vitro (Fig. 3). One is composed of two ligated oligonucleotides, which we will refer

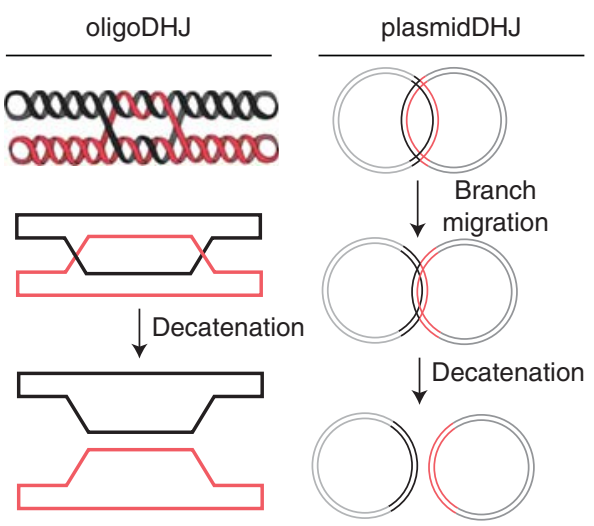

Figure 3. DHJ substrates. Several substrates have been used to study $\mathrm{HJ}$ dissolution in vitro. The oligoDHJ substrate (left) is generated by the annealing and ligation of two oligonucleotides, leading to the formation of two $\mathrm{HJ}$ separated by 14 bp of quasi-homology. The processing of this substrate by the dissolvasome requires little or no branch migration allowing the investigation of the decatenation step. Following dissolution, the two strands forming the oligoDHJ substrate are physically separated and remain circular. The plasmidDHJ (right) comprises two circular dsDNA molecules associated though a region of homology (depicted in black and red). This substrate permits the analysis of the entire dissolution reaction, which in this case will lead to the complete separation of the two plasmids. 
to as the oligoDHJ. This molecule can be viewed as the product of convergent branch migration at or near the point where decatenation can occur, allowing analysis of the decatenation step (Fu et al. 1994; Wu and Hickson 2003). Although this substrate is quite easy to prepare (Bachrati and Hickson 2009), it does not contain HJs that can migrate freely and, therefore, does not permit the biochemical analysis of the convergent branch migration step of dissolution. By contrast, the second, much larger substrate (termed plasmidDHJ) does contain two migratable HJs but is technically much more challenging to prepare (Plank and Hsieh 2006; Chen et al. 2013). The lack of a facile method for the preparation of a substrate containing two fully branch migratable HJs represents one important difficulty with conducting biochemical analysis of the $\mathrm{dHJ}$ dissolution reaction in vitro.

\section{Importance of Protein Complex Formation for Dissolution}

The genetic data reviewed above suggested that the physical interaction between the helicase and the topoisomerase component of the dissolvasome would be crucial for the dissolution reaction in vivo. Consistent with this, highly efficient dissolution is observed in vitro only when BLM or Sgs1 are incubated in the presence of the topoisomerase III enzyme from the same species. Moreover, the BLM/Sgs1 component cannot be replaced by another RecQ helicase (Wu and Hickson 2003; Wu et al. 2005; Plank et al. 2006; Raynard et al. 2006; Cejka et al. 2010). However, a BLM mutant lacking the first 212 residues shown to be responsible for interaction with TopoIII $\alpha$ is as efficient as the full-length BLM in catalyzing dissolution of the oligoDHJ substrate ( $\mathrm{Wu}$ et al. 2005). Together, these data indicate that BLM/TopoIII $\alpha$ physical interaction is not absolutely required for dissolution in vitro. Consistent with this, the reaction can occur, albeit quite inefficiently, when BLM/Sgs1 is utilized together with a noncognate type IA topoisomerase ( $\mathrm{Wu}$ et al. 2006). Hence, beside their ability to interact together, BLM/Sgs1 and type IA topoisomer- ases must exhibit specific properties that allow them to catalyze the different steps leading to HJ dissolution.

\section{The Decatenation Step}

The hemicatenated DNA structure generated by the fusion/collapse of two HJs is the key intermediate in $\mathrm{dHJ}$ dissolution. Although the actual structure of two fused HJs is not known, it is likely to correspond to a combination of one or more single-stranded catenanes (Fig. 1B). Among all topoisomerases, only type IA topoisomerases can perform the decatenation reaction required for the topological separation of the two duplexes (Wu and Hickson 2003; Plank et al. 2006; Wu et al. 2006; Cejka et al. 2010). This specificity has been attributed to the unique ability of type IA topoisomerases to catalyze efficient single stranded decatenation (Brown and Cozzarelli 1981; Harmon et al. 1999; Yang et al. 2010; Cejka et al. 2012). The specific requirement of a type IA topoisomerase during the last step of $\mathrm{HJ}$ dissolution may also reside in their affinity for complex DNA structures. Indeed, both yTop3 and hTopoIII $\alpha$ exhibit remarkable affinity for $\mathrm{HJ}$-containing substrates that are largely double stranded in character (Fig. 3) (Wu et al. 2006; Chen and Brill 2007). This is surprising because catalysis by the type IA topoisomerase requires the presence of single-stranded DNA (ssDNA) in the substrate (Srivenugopal et al. 1984; Kirkegaard and Wang 1985; Kim and Wang 1992; Chen and Brill 2007). Type IA topoisomerases may be viewed, therefore, as DNA structure-selective topoisomerases, making them ideally suited for the processing of complex DNA structures.

\section{Function of the RecQ Helicase during Decatenation}

Despite the above discussion, the type IA topoisomerase cannot perform efficient decatenation of a $\mathrm{dHJ}$ alone, because this requires the presence of the RecQ helicase. Moreover, the helicase likely plays an active role during decatenation as evidenced by the requirement of 
Dissolution of Double Holliday Junctions

its ATPase activity for the reaction ( $\mathrm{Wu}$ and Hickson 2003; Raynard et al. 2006; Bussen et al. 2007; Cejka et al. 2010; Yang et al. 2010; Chen et al. 2014). It is likely that the RecQ unwinding activity remodels the substrate into a conformation that can be efficiently processed by the type IA topoisomerase-for instance, by providing accessible ssDNA. Indeed, Sgs1/ BLM helicases are known to stimulate both the relaxation and decatenation activities of Top3/TopoIII $\alpha$ (Wu and Hickson 2002; Yang et al. 2010; Cejka et al. 2012). However, none of the other RecQ helicases tested thus far can assist the type IA topoisomerase during DHJ decatenation (Wu and Hickson 2003; Wu et al. 2005; Plank et al. 2006; Cejka et al. 2010). This observation suggests that the Sgs1/BLM subfamily might possess functional domain(s) that are missing from or altered in other RecQ helicases and that are crucial during dissolution. For instance, the unique HRDC domain of BLM is absolutely required for dissolution (Bernstein and Keck 2005; Wu et al. 2005). This domain displays specific DNA binding properties allowing BLM to specifically bind to and unwind a HJ (Janscak et al. 2003; Wu et al. 2005). More important, amino-terminal extensions of several RecQ helicases are known to carry additional activities providing a functional specialization (Fig. 2) (Huang et al. 1998; Matsuno et al. 2006). Although the extreme amino-terminal region of BLM/Sgs1 where TopoIII $\alpha /$ Top3 binds is dispensable for efficient dissolution of the oligoDHJ substrate (Wu et al. 2005), a function for the extended amino-terminal region during catalysis of convergent branch migration should not be excluded.

\section{Convergent Branch Migration Step}

If the type IA topoisomerase surely provides the crucial catalytic activity during decatenation, the RecQ helicase is certainly the central catalytic component of the dissolvasome during convergent branch migration. Indeed, many RecQ helicases, including BLM and Sgs1, exhibit efficient branch migration activities on a single HJ (Constantinou et al. 2000; Karow et al.
2000; Bugreev et al. 2008; Cejka and Kowalczykowski 2010). The mechanism of this branch migration activity is unknown, but it might result from the association of the unwinding and annealing activities of RecQ helicases (Cheok et al. 2005). More important, besides the fact that many RecQ helicases can catalyze single HJ branch migration, thus far only the Sgs1/ BLM subtype has a proven role during $\mathrm{dHJ}$ dissolution. This specificity highlights once again the potential existence of atypical properties shared only by Sgs1 and BLM. For example, the substrate specificity provided by the poorly conserved HRDC domain of the BLM/ Sgs1 helicases could play a crucial function during convergent branch migration ( $\mathrm{Wu}$ et al. 2005). An alternative model, yet to be demonstrated, proposes that the RecQ core domain simply functions in the translocation of the protein along ssDNA, whereas it is the aminoterminal domain that catalyzes branch migration via a strand exchange activity (Chen and Brill 2010).

Function of the Type IA Topoisomerase during Convergent Branch Migration

In the context of a topologically closed $\mathrm{dHJ}$ (exemplified by the plasmidDHJ substrate), each strand of a duplex is associated with its complementary strand in the other duplex through base pairing and topological linking (Fig. 3). Therefore, the initial step of $\mathrm{dHJ}$ dissolution cannot be catalyzed by a helicase alone; instead, it requires the combined action of a helicase and a topoisomerase to permit the two HJs to converge. According to the twin supercoiling domain model, branch migration catalyzed by a helicase will lead to the redistribution of topological links, with an accumulation of positive supercoils between the two junctions and negative supercoils behind them (Liu and Wang 1987; Plank et al. 2006). These torsional stresses must be dissipated by the action of a topoisomerase. Indeed, in vitro, Sgs1 together with wheat germ TopoIB, which exhibits an efficient relaxation activity of both positive and negative supercoils, can catalyze convergent branch migration of the plasmidDHJ to generate products 
corresponding to a nearly or completely fused HJ (Cejka et al. 2010). However, the final decatenation step still requires a type IA topoisomerase. This observation indicates that, at least in vitro, Sgs1 does not require the presence of its Top3 partner to catalyze convergent branch migration, and that the dissipation of the topological stress generated during convergent branch migration can occur through an "uncoupled" mechanism. Having said that, as a component of the same complex, the type IA topoisomerase subunit of the dissolvasome likely supports this function in vivo. Consistent with this, when assayed on the plasmidDHJ substrate, the association of Sgs1 or D. melanogaster BLM with its cognate type IA topoisomerase formed a proficient branch migrating machine (Plank et al. 2006; Cejka et al. 2010). The precise mechanism by which Top3 assists the efficient convergent branch migration catalyzed by Sgs1 in a topologically closed substrate remains unclear, mainly because of the well-established inefficiency of type IA topoisomerases to relax either negative or positive supercoils. However, one must consider that the affinity of type IA topoisomerases for a four-way junction, as well as its association with a cognate RecQ helicase, would allow its positioning in the vicinity of the $\mathrm{HJ}$ and provide the ssDNA necessary for relaxation of torsional stress (Cejka et al. 2012). One could also speculate that the type IA topoisomerase directly manipulates the topology of the HJ itself, allowing the branch migration to occur without generating torsional stress (Duguet 1997; Plank and Hsieh 2009).

In the previous paragraphs, we have attempted to describe the individual contribution of the two catalytic subunits of the dissolvasome during the convergent branch migration and decatenation steps of dissolution. However, the dissolution reaction appears to result not only from the simple addition of two individual activities, but also from a potential intricate cooperation of these activities, emphasizing the complex nature of dissolution reaction. This view is further strengthened by the presence and the function of the third component of the minimal dissolvasome core complex, RMI1.

\section{RMI1}

\section{Rmi1, an Integral Component of the Core Dissolvasome}

In $S$. cerevisae, two independent approaches led to the identification of Rmil as a candidate protein functioning alongside Sgs 1 and Top3 in the maintenance of genomic stability (Bellaoui et al. 2003; Tong et al. 2004). rmil mutants phenocopy all investigated $\Delta t o p 3$-associated phenotypes including slow growth, sensitivity to DNA damaging agents, an increased rate of mitotic recombination, meiotic defects, gross chromosomal rearrangements, and an accumulation of HR intermediates (Chang et al. 2005; Mullen et al. 2005; Ashton et al. 2011). Moreover, $\Delta$ rmil is synthetically lethal with mutations in genes encoding putative HJ resolvases, and this synthetic lethality can be suppressed by inactivation of early steps of HR. Finally, rmil is epistatic to top3, and the slow growth phenotype of $\Delta r m i 1$ strains can be suppressed by mutations in the SGS1 gene (Chang et al. 2005; Mullen et al. 2005). Rmil is a conserved protein, being present in nearly all eukaryotes although no ortholog has been found in D. melanogaster (Yin et al. 2005; Chen et al. 2012). As with TopoIII $\alpha$, Rmil homologs are essential in S. pombe, A. thaliana, and mice (Chang et al. 2005; Hartung et al. 2008; Chen et al. 2011; Guiraldelli et al. 2013). Finally, in HeLa cells, depletion of RMI1 by siRNA is associated with an increased level of SCE (Yin et al. 2005).

The confirmation that Rmil is an integral and conserved component of the dissolvasome came with the finding that the human homolog of yeast Rmi1, RMI1 (originally designated BLAP75, a BLM-associated protein of $75 \mathrm{kDa}$ ) forms a stable complex with both BLM and TopoIII $\alpha$ in vivo and in vitro (Meetei et al. 2003; Yin et al. 2005; Raynard et al. 2006). Similarly, Rmil was also found in a complex with Sgs1 and Top3 in yeast (Chang et al. 2005; Mullen et al. 2005; Chen and Brill 2007). Although Rmil/RMI1 seems to interact independently with Sgs1/BLM and Top3/TopoIII $\alpha$, it forms a stable heterodimer with the type IA topoisomerase. In contrast, it appears that at least a proportion of the RecQ helicase is found outside of 
the apparently obligate Rmi1/Top3 complex in cells (Mullen et al. 2005; Chen and Brill 2007).

\section{Role of RMI1 during HJ Dissolution}

RMI1 strongly stimulates the dissolution reaction catalyzed by BLM and TopoIII $\alpha$, and this stimulation requires Rmilinteraction with BLM and TopoIII $\alpha$ (Raynard et al. 2006, 2008; Wu et al. 2006; Cejka et al. 2010). Importantly, the stimulatory effect of RMI1 on dissolution is so impressive that the reaction catalyzed by TopoIII $\alpha$ and BLM alone appears extremely inefficient by comparison. RMI1 is, therefore, considered to be a key component of the efficient dissolvasome.

Human RMI1 is composed of two OB-fold domains linked together by an undefined domain (Fig. 2). However, only the amino-terminal domain of hRMI1 is conserved in yeast. This conserved OB-fold domain is required for TopoIII $\alpha$ and BLM binding and is sufficient for efficient dissolution in vitro (Raynard et al. 2008). As a consequence, the conserved dissolvasome core complex can be considered as being composed of TopoIII $\alpha$, BLM, together with the conserved amino-terminal domain of RMI1.

Beside its ability to physically associate with BLM and TopoIII $\alpha$, RMI1 does not seem to exhibit any obvious biochemical property that would readily explain its contribution to dissolution. Because RMI1 is an OB fold containing protein (Fig. 2), it is tempting to speculate that it is a DNA binding protein (Murzin 1993). RMI1 does exhibit some affinity for DNA that can only be observed with high protein concentrations or with cross-linking treatment (Mullen et al. 2005; Wu et al. 2006; Chen and Brill 2007; Xu et al. 2008; Wang et al. 2010). Thus, it is unlikely that its role in dissolution depends entirely on its apparently unimpressive DNA binding properties. Moreover, the putative DNA binding domain of RMI1 has been shown to correspond to the nonconserved carboxy-terminal OB-fold domain, dispensable for dissolution stimulation of the human BLM/TopoIII $\alpha$ decatenation activity (Fig. 2) (Raynard et al. 2008). The role of the Rmil subunit during dissolution is mainly attributed to its direct influence on TopoIII $\alpha$ /
Top3 biochemical properties. Indeed, Rmil/ RMI1 proteins have been shown to significantly enhance Top3/TopoIII $\alpha$ binding to different substrates including the $\mathrm{dHJ}$ and ssDNA ( $\mathrm{Wu}$ et al. 2006; Chen and Brill 2007) and to influence several activities of topoisomerase III enzymes (Chen and Brill 2007; Yang et al. 2010; Cejka et al. 2012). Importantly, in each case, the effect of Rmil/RMI1 on Top3/TopoIII $\alpha$ activity is synergistically enhanced in the presence of Sgs1/BLM, indicating cooperation between RMI1 and BLM in the stimulation of the topoisomerase (Wu et al. 2006; Yang et al. 2010, 2012; Cejka et al. 2012).

Analysis of the effect of RMI1 on the dissolution of the plasmidDHJ substrate indicated that Rmil functions primarily in the decatenation step of dissolution, playing little or no role during convergent branch migration (Cejka et al. 2010). However, because the TopoIII $\alpha /$ RMI1 subcomplex strongly stimulates BLM unwinding activity of a four-way junction, an involvement of TopoIII $\alpha /$ RMI1 during the branch migration process is possible (Bussen et al. 2007; Raynard et al. 2008). This stimulation requires only the conserved amino-terminal portion of RMI1 (Fig. 2), suggesting that the stimulation of the RecQ helicase unwinding activity by the Topoisomerase/Rmil subcomplex might be a conserved feature during evolution (Raynard et al. 2008). The synergistic influence exerted by Sgs1/BLM and Top3/TopoIII $\alpha$ on each other in the presence of Rmil/RMI1 emphasizes the highly sophisticated reaction required for efficient HJ dissolution. In this context, the Rmil subunit might act as both the architect and the choreographer of the dissolvasome, being responsible for the tight association and coordination between the two catalytical subunits. Indeed, recent biochemical and structural data strongly suggest that Rmil regulates the decatenation function of Top3 thoroughly altering the dynamics of the opening and closing of the topoisomerase gate (Cejka et al. 2012; Bocquet et al. 2014).

An Extended Human Dissolvasome Complex

Efficient dissolution of the more complex plasmidDHJ substrate also seems to require the 
presence of the ssDNA binding protein RPA (Plank et al. 2006; Cejka et al. 2010). This stimulatory effect is attributed to the binding to and stimulation of BLM/Sgs1 helicase activity by RPA, suggesting that RPA could be part of an extended dissolvasome complex (Brosh et al. 2000; Meetei et al. 2003; Doherty et al. 2005; Hegnauer et al. 2012). This proposal is supported by recent findings showing that the stimulatory effect of hRPA on $\mathrm{dHJ}$ dissolution requires both its ssDNA binding activity and a direct physical interaction with RMI1 (Xue et al. 2013). Importantly, however, the hRPA interacting domain of RMI1 resides in the nonconserved central region of hRMI1, suggesting that the physical integration of RPA within the dissolvasome might not be conserved in yeast (Xue et al. 2013). Consistently, yRPA and E. coli single-strand binding protein (SSB) stimulate dissolution by yeast Sgs1/Top3/Rmil complex to a similar degree (Cejka et al. 2010). hRPA is not the only protein found to physically associate with the carboxy-terminal extension found in higher eukaryotic RMI1. Indeed, this nonconserved region is responsible for the association of the dissolvasome complex with FANCM and RMI2 (Deans and West 2009; Yang et al. 2010; Hoadley et al. 2012; Manthei and Keck 2013). Among them, only RMI2 is thought to be important for the functionality of the complex in vivo (Fig. 2) (Singh et al. 2008; Xu et al. 2008; Hoadley et al. 2010). However, this additional $\mathrm{OB}$ fold containing protein has a minor effect on the efficiency of dissolution in vitro (Singh et al. 2008; Xu et al. 2008).

\section{CONCLUDING REMARKS}

Ten years after its discovery, the precise mechanism by which the dissolvasome catalyzes dissolution still remains enigmatic. Indeed, despite its apparent simplicity, dissolution clearly requires some remarkably complicated biochemical cooperation between a type IA topoisomerase, a RecQ helicase, and an OB-fold protein. Many aspects of the process remain elusive and will deserve attention in the future. For instance, the reasons underlying the apparent specialization of one subgroup of RecQ hel- icases defined by Sgs1/BLM in dissolution and the mechanical contribution of Type IA topoisomerase during convergent branch migration are still unknown. In addition, several structural elements might be illuminating to understand the architecture of the complex. It is noteworthy that the dissolvasome complex is thought to dissipate other DNA structures such as late replication intermediates or catenated DNA (Wu et al. 1999; Mankouri and Hickson 2007; Suski and Marians 2008; Cejka et al. 2012; Manthei and Keck 2013). Because the mechanism underlying those processes and dissolution might be similar, it would also be informative to carry on mechanistic studies of those reactions.

\section{ACKNOWLEDGMENTS}

We thank Drs. Hocine Mankouri and Kata Sarlos for helpful comments on the manuscript. Work in the authors' laboratory is supported by the Nordea Foundation, The Villum Kann Rasmussen Fund, The European Research Council, The Danish Medical Research Council, and The Danish Cancer Society.

\section{REFERENCES}

\section{${ }^{*}$ Reference is also in this subject collection.}

Andersen SL, Kuo HK, Savukoski D, Brodsky MH, Sekelsky J. 2011. Three structure-selective endonucleases are essential in the absence of BLM helicase in Drosophila. PLoS Genet 7: e1002315.

Ashton TM, Mankouri HW, Heidenblut A, McHugh PJ, Hickson ID. 2011. Pathways for Holliday junction processing during homologous recombination in Saccharomyces cerevisiae. Mol Cell Biol 31: 1921-1933.

Bachrati CZ, Hickson ID. 2003. RecQ helicases: Suppressors of tumorigenesis and premature aging. Biochem J 374: 577-606.

Bachrati CZ, Hickson ID. 2009. Dissolution of double Holliday junctions by the concerted action of BLM and topoisomerase III $\alpha$. Methods Mol Biol 582: 91-102.

Bastin-Shanower SA, Fricke WM, Mullen JR, Brill SJ. 2003. The mechanism of Mus81-Mms4 cleavage site selection distinguishes it from the homologous endonuclease Rad1-Rad10. Mol Cell Biol 23: 3487-3496.

Bellaoui M, Chang M, Ou J, Xu H, Boone C, Brown GW. 2003. Elg1 forms an alternative RFC complex important for DNA replication and genome integrity. $E M B O J$ 22: 4304-4313. 
Dissolution of Double Holliday Junctions

Bennett RJ, Noirot-Gros MF, Wang JC. 2000. Interaction between yeast Sgs1 helicase and DNA topoisomerase III. J Biol Chem 275: 26898-26905.

Bernstein DA, Keck JL. 2005. Conferring substrate specificity to DNA helicases: Role of the RecQ HRDC domain. Structure 13: 1173-1182.

Bernstein KA, Gangloff S, Rothstein R. 2010. The RecQ DNA helicases in DNA repair. Annu Rev Genet 44: 393 417.

Bocquet N, Bizard AH, Abdulrahman W, Larsen NB, Faty M, Cavadini S, Bunker RD, Kowalczykowski SC, Cejka P, Hickson ID, et al. 2014. Structural and mechanistic insight into Holliday-junction dissolution by Topoisomerase III $\alpha$ and RMI1. Nat Struct Mol Biol 2121: 261-268.

Boddy MN, Lopez-Girona A, Shanahan P, Interthal H, Heyer WD, Russell P. 2000. Damage tolerance protein Mus81 associates with the FHA1 domain of checkpoint kinase Cds1. Mol Cell Biol 20: 8758-8766.

Brosh RM Jr., Li JL, Kenny MK, Karow JK, Cooper MP, Kureekattil RP, Hickson ID, Bohr VA. 2000. Replication protein A physically interacts with the Bloom's syndrome protein and stimulates its helicase activity. J Biol Chem 275: $23500-23508$.

Brown PO, Cozzarelli NR. 1981. Catenation and knotting of duplex DNA by type 1 topoisomerases: A mechanistic parallel with type 2 topoisomerases. Proc Natl Acad Sci 78: 843-847.

Bugreev DV, Brosh RM Jr., Mazin AV. 2008. RECQ1 possesses DNA branch migration activity. J Biol Chem 283: 20231-20242.

Bussen W, Raynard S, Busygina V, Singh AK, Sung P. 2007. Holliday junction processing activity of the BLM-Topo III $\alpha$-BLAP75 complex. J Biol Chem 282: 31484-31492.

Bzymek M, Thayer NH, Oh SD, Kleckner N, Hunter N. 2010. Double Holliday junctions are intermediates of DNA break repair. Nature 464: 937-941.

Carotenuto W, Liberi G. 2010. Mitotic inter-homologue junctions accumulate at damaged DNA replication forks in recQ mutants. DNA Repair 9: 661-669.

Cejka P, Kowalczykowski SC. 2010. The full-length Saccharomyces cerevisiae Sgs 1 protein is a vigorous DNA helicase that preferentially unwinds Holliday junctions. J Biol Chem 285: 8290-8301.

Cejka P, Plank JL, Bachrati CZ, Hickson ID, Kowalczykowski SC. 2010. Rmil stimulates decatenation of double Holliday junctions during dissolution by Sgs1-Top3. Nat Struct Mol Biol 17: 1377-1382.

Cejka P, Plank JL, Dombrowski CC, Kowalczykowski SC. 2012. Decatenation of DNA by the S. cerevisiae Sgs1Top3-Rmil and RPA complex: A mechanism for disentangling chromosomes. Mol Cell 47: 886-896.

Chaganti RS, Schonberg S, German J. 1974. A manyfold increase in sister chromatid exchanges in Bloom's syndrome lymphocytes. Proc Natl Acad Sci 71: 4508-4512.

Chakraverty RK, Kearsey JM, Oakley TJ, Grenon M, de La Torre Ruiz MA, Lowndes NF, Hickson ID. 2001. Topoisomerase III acts upstream of Rad53p in the $S$-phase DNA damage checkpoint. Mol Cell Biol 21: 7150-7162.

Champoux JJ. 2001. DNA topoisomerases: Structure, function, and mechanism. Annu Rev Biochem 70: 369-413.
Chang M, Bellaoui M, Zhang C, Desai R, Morozov P, Delgado-Cruzata L, Rothstein R, Freyer GA, Boone C, Brown GW. 2005. RMI1/NCE4, a suppressor of genome instability, encodes a member of the RecQ helicase/Topo III complex. EMBO J 24: 2024-2033.

Chen CF, Brill SJ. 2007. Binding and activation of DNA topoisomerase III by the Rmil subunit. J Biol Chem 282: 28971-28979.

Chen CF, Brill SJ. 2010. An essential DNA strand-exchange activity is conserved in the divergent N-termini of BLM orthologs. EMBO J 29: 1713-1725.

Chen H, You MJ, Jiang Y, Wang W, Li L. 2011. RMI1 attenuates tumor development and is essential for early embryonic survival. Mol Carcinog 50: 80-88.

Chen SH, Wu CH, Plank JL, Hsieh TS. 2012. Essential functions of C terminus of Drosophila Topoisomerase III $\alpha$ in double Holliday junction dissolution. J Biol Chem 287: 19346-19353.

Chen SH, Plank JL, Willcox S, Griffith JD, Hsieh TS. 2013. Improved methods for creating migratable Holliday junction substrates. Nucleic Acids Res 41: e60.

Chen SH, Plank JL, Willcox S, Griffith JD, Hsieh TS. 2014. Top $3 \alpha$ is required during the convergent migration step of double Holliday junction dissolution. PloS ONE 9: e83582.

Cheok CF, Wu L, Garcia PL, Janscak P, Hickson ID. 2005. The Bloom's syndrome helicase promotes the annealing of complementary single-stranded DNA. Nucleic Acids Res 33: 3932-3941.

Chu WK, Hickson ID. 2009. RecQ helicases: Multifunctional genome caretakers. Nat Rev Cancer 9: 644-654.

Constantinou A, Tarsounas M, Karow JK, Brosh RM, Bohr VA, Hickson ID, West SC. 2000. Werner's syndrome protein (WRN) migrates Holliday junctions and co-localizes with RPA upon replication arrest. EMBO Rep 1: 80-84.

Deans AJ, West SC. 2009. FANCM connects the genome instability disorders Bloom's syndrome and Fanconi anemia. Mol Cell 36: 943-953.

Doe CL, Ahn JS, Dixon J, Whitby MC. 2002. Mus81-Eme1 and Rqh1 involvement in processing stalled and collapsed replication forks. J Biol Chem 277: 32753-32759.

Doherty KM, Sommers JA, Gray MD, Lee JW, von Kobbe C, Thoma NH, Kureekattil RP, Kenny MK, Brosh RM Jr. 2005. Physical and functional mapping of the replication protein interaction domain of the Werner and Bloom syndrome helicases. J Biol Chem 280: 29494-29505.

Duguet M. 1997. When helicase and topoisomerase meet! J Cell Sci 110: 1345-1350.

Duno M, Thomsen B, Westergaard O, Krejci L, Bendixen C. 2000. Genetic analysis of the Saccharomyces cerevisiae Sgs1 helicase defines an essential function for the Sgs1Top 3 complex in the absence of SRS2 or TOP1. Mol Gen Genet 264: 89-97.

Fabre F, Chan A, Heyer WD, Gangloff S. 2002. Alternate pathways involving Sgs1/Top3, Mus81/ Mms4, and Srs2 prevent formation of toxic recombination intermediates from single-stranded gaps created by DNA replication. Proc Natl Acad Sci 99: 16887-16892.

Forterre P, Gadelle D. 2009. Phylogenomics of DNA topoisomerases: Their origin and putative roles in the emer- 
A.H. Bizard and I.D. Hickson

gence of modern organisms. Nucleic Acids Res 37: 679692.

Fricke WM, Kaliraman V, Brill SJ. 2001. Mapping the DNA topoisomerase III binding domain of the Sgs1 DNA helicase. J Biol Chem 276: 8848-8855.

Fu TJ, Tse-Dinh YC, Seeman NC. 1994. Holliday junction crossover topology. J Mol Biol 236: 91-105.

Gangloff S, McDonald JP, Bendixen C, Arthur L, Rothstein R. 1994. The yeast type I topoisomerase Top3 interacts with Sgs1, a DNA helicase homolog: A potential eukaryotic reverse gyrase. Mol Cell Biol 14: 8391-8398.

Gangloff S, de Massy B, Arthur L, Rothstein R, Fabre F. 1999. The essential role of yeast topoisomerase III in meiosis depends on recombination. EMBO J 18: 1701-1711.

German J. 1993. Bloom syndrome: A Mendelian prototype of somatic mutational disease. Medicine 72: 393-406.

Goodwin A, Wang SW, Toda T, Norbury C, Hickson ID. 1999. Topoisomerase III is essential for accurate nuclear division in Schizosaccharomyces pombe. Nucleic Acids Res 27: 4050-4058.

Guiraldelli MF, Eyster C, Pezza RJ. 2013. Genome instability and embryonic developmental defects in RMI1 deficient mice. DNA Repair 12: 835-843.

Harmon FG, DiGate RJ, Kowalczykowski SC. 1999. RecQ helicase and topoisomerase III comprise a novel DNA strand passage function: A conserved mechanism for control of DNA recombination. Mol Cell 3: 611-620.

Hartung F, Suer S, Puchta H. 2007. Two closely related RecQ helicases have antagonistic roles in homologous recombination and DNA repair in Arabidopsis thaliana. Proc Natl Acad Sci 104: 18836-18841.

Hartung F, Suer S, Knoll A, Wurz-Wildersinn R, Puchta H. 2008. Topoisomerase $3 \alpha$ and RMI1 suppress somatic crossovers and are essential for resolution of meiotic recombination intermediates in Arabidopsis thaliana. PLoS Genet 4: e1000285.

Hegnauer AM, Hustedt N, Shimada K, Pike BL, Vogel M, Amsler P, Rubin SM, van Leeuwen F, Guenole A, van Attikum H, et al. 2012. An N-terminal acidic region of Sgs1 interacts with Rpa70 and recruits Rad53 kinase to stalled forks. EMBO J 31: 3768-3783.

Hoadley KA, Xu D, Xue Y, Satyshur KA, Wang W, Keck JL. 2010. Structure and cellular roles of the RMI core complex from the Bloom syndrome dissolvasome. Structure 18: 1149-1158.

Hoadley KA, Xue Y, Ling C, Takata M, Wang W, Keck JL. 2012. Defining the molecular interface that connects the Fanconi anemia protein FANCM to the Bloom syndrome dissolvasome. Proc Natl Acad Sci 109: 4437-4442.

Hu P, Beresten SF, van Brabant AJ, Ye TZ, Pandolfi PP, Johnson FB, Guarente L, Ellis NA. 2001. Evidence for BLM and Topoisomerase III $\alpha$ interaction in genomic stability. Hum Mol Genet 10: 1287-1298.

Huang S, Li B, Gray MD, Oshima J, Mian IS, Campisi J. 1998. The premature ageing syndrome protein, WRN, is a $3^{\prime} \rightarrow 5^{\prime}$ exonuclease. Nat Genet 20: $114-116$.

Ira G, Malkova A, Liberi G, Foiani M, Haber JE. 2003. Srs2 and Sgs1-Top3 suppress crossovers during double-strand break repair in yeast. Cell 115: 401-411.

Janscak P, Garcia PL, Hamburger F, Makuta Y, Shiraishi K, Imai Y, Ikeda H, Bickle TA. 2003. Characterization and mutational analysis of the RecQ core of the bloom syndrome protein. J Mol Biol 330: 29-42.

Kaliraman V, Mullen JR, Fricke WM, Bastin-Shanower SA, Brill SJ. 2001. Functional overlap between Sgs1-Top3 and the Mms4-Mus81 endonuclease. Genes Dev 15: 27302740.

Karow JK, Constantinou A, Li JL, West SC, Hickson ID. 2000. The Bloom's syndrome gene product promotes branch migration of Holliday junctions. Proc Natl Acad Sci 97: 6504-6508.

Kim RA, Wang JC. 1992. Identification of the yeast TOP3 gene product as a single strand-specific DNA topoisomerase. J Biol Chem 267: 17178-17185.

Kirkegaard K, Wang JC. 1985. Bacterial DNA topoisomerase I can relax positively supercoiled DNA containing a single-stranded loop. J Mol Biol 185: 625-637.

Krejci L, Altmannova V, Spirek M, Zhao X. 2012. Homologous recombination and its regulation. Nucleic Acids Res 40: $5795-5818$.

Kusano K, Berres ME, Engels WR. 1999. Evolution of the RECQ family of helicases: A Drosophila homolog, Dmblm, is similar to the human Bloom syndrome gene. Genetics 151: 1027-1039.

LaRocque JR, Stark JM, Oh J, Bojilova E, Yusa K, Horie K, Takeda J, Jasin M. 2011. Interhomolog recombination and loss of heterozygosity in wild-type and Bloom syndrome helicase (BLM)-deficient mammalian cells. Proc Natl Acad Sci 108: 11971-11976.

Larsen NB, Hickson ID. 2013. RecQ helicases: Conserved guardians of genomic integrity. Adv Exp Med Biol 767: $161-184$.

Laursen LV, Bjergbaek L, Murray JM, Andersen AH. 2003. RecQ helicases and topoisomerase III in cancer and aging. Biogerontology 4: 275-287.

Li W, Wang JC. 1998. Mammalian DNA topoisomerase III $\alpha$ is essential in early embryogenesis. Proc Natl Acad Sci 95: 1010-1013.

Liberi G, Maffioletti G, Lucca C, Chiolo I, Baryshnikova A, Cotta-Ramusino C, Lopes M, Pellicioli A, Haber JE, Foiani M. 2005. Rad51-dependent DNA structures accumulate at damaged replication forks in sgs 1 mutants defective in the yeast ortholog of BLM RecQ helicase. Genes Dev 19: 339-350.

Liu LF, Wang JC. 1987. Supercoiling of the DNA template during transcription. Proc Natl Acad Sci 84: 7024-7027.

Luo G, Santoro IM, McDaniel LD, Nishijima I, Mills M, Youssoufian H, Vogel H, Schultz RA, Bradley A. 2000. Cancer predisposition caused by elevated mitotic recombination in Bloom mice. Nat Genet 26: 424-429.

Maftahi M, Han CS, Langston LD, Hope JC, Zigouras N, Freyer GA. 1999. The top ${ }^{+}$gene is essential in Schizosaccharomyces pombe and the lethality associated with its loss is caused by Rad 12 helicase activity. Nucleic Acids Res 27: 4715-4724.

Mankouri HW, Hickson ID. 2006. Top3 processes recombination intermediates and modulates checkpoint activity after DNA damage. Mol Biol Cell 17: 4473-4483.

Mankouri HW, Hickson ID. 2007. The RecQ helicase-topoisomerase III-Rmil complex: A DNA structure-specific “dissolvasome?" Trends Biochem Sci 32: 538-546. 
Dissolution of Double Holliday Junctions

Mankouri HW, Ashton TM, Hickson ID. 2011. Holliday junction-containing DNA structures persist in cells lacking Sgs1 or Top3 following exposure to DNA damage. Proc Natl Acad Sci 108: 4944-4949.

Manthei KA, Keck JL. 2013. The BLM dissolvasome in DNA replication and repair. Cell Mol Life Sci 70: 4067-4084.

Matsuno K, Kumano M, Kubota Y, Hashimoto Y, Takisawa H. 2006. The N-terminal noncatalytic region of Xenopus RecQ4 is required for chromatin binding of DNA polymerase $\alpha$ in the initiation of DNA replication. Mol Cell Biol 26: 4843-4852.

Meetei AR, Sechi S, Wallisch M, Yang D, Young MK, Joenje H, Hoatlin ME, Wang W. 2003. A multiprotein nuclear complex connects Fanconi anemia and Bloom syndrome. Mol Cell Biol 23: 3417-3426.

* Mehta A, Haber JE. 2014. Sources of DNA double-strand breaks and models for recombinational DNA repair. Cold Spring Harb Perspect Biol doi: 10.1101/cshperspect .a016428.

Mullen JR, Kaliraman V, Brill SJ. 2000. Bipartite structure of the SGS1 DNA helicase in Saccharomyces cerevisiae. Genetics 154: 1101-1114.

Mullen JR, Kaliraman V, Ibrahim SS, Brill SJ. 2001. Requirement for three novel protein complexes in the absence of the Sgs1 DNA helicase in Saccharomyces cerevisiae. Genetics 157: 103-118.

Mullen JR, Nallaseth FS, Lan YQ, Slagle CE, Brill SJ. 2005. Yeast Rmil/Nce4 controls genome stability as a subunit of the Sgs1-Top3 complex. Mol Cell Biol 25: 4476-4487.

Murzin AG. 1993. OB(oligonucleotide/oligosaccharide binding)-fold: Common structural and functional solution for non-homologous sequences. EMBO J 12: 861 867.

Myung K, Datta A, Chen C, Kolodner RD. 2001. SGS1, the Saccharomyces cerevisiae homologue of BLM and WRN, suppresses genome instability and homeologous recombination. Nat Genet 27: 113-116.

Myung K, Kolodner RD. 2002. Suppression of genome instability by redundant S-phase checkpoint pathways in Saccharomyces cerevisiae. Proc Natl Acad Sci 99: 45004507.

Nakayama H. 2005. Escherichia coli RecQ helicase: A player in thymineless death. Mutat Res 577: 228-236.

Nasmyth KA. 1982. Molecular genetics of yeast mating type. Annu Rev Genet 16: 439-500.

Oakley TJ, Goodwin A, Chakraverty RK, Hickson ID. 2002. Inactivation of homologous recombination suppresses defects in topoisomerase III-deficient mutants. DNA Repair 1: 463-482.

Oh M, Choi IS, Park SD. 2002. Topoisomerase III is required for accurate DNA replication and chromosome segregation in Schizosaccharomyces pombe. Nucleic Acids Res 30: 4022-4031.

Onoda F, Seki M, Miyajima A, Enomoto T. 2000. Elevation of sister chromatid exchange in Saccharomyces cerevisiae sgsl disruptants and the relevance of the disruptants as a system to evaluate mutations in Bloom's syndrome gene. Mutat Res 459: 203-209.

Onodera R, Seki M, Ui A, Satoh Y, Miyajima A, Onoda F, Enomoto T. 2002. Functional and physical interaction between Sgs1 and Top3 and Sgs1-independent function of Top3 in DNA recombination repair. Genes Genetic Syst 77: $11-21$.

Plank JL, Hsieh TS. 2006. A novel, topologically constrained DNA molecule containing a double Holliday junction: Design, synthesis, and initial biochemical characterization. J Biol Chem 281: 17510-17516.

Plank J, Hsieh TS. 2009. Helicase-appended topoisomerases: New insight into the mechanism of directional strand transfer. J Biol Chem 284: 30737-30741.

Plank JL, Chu SH, Pohlhaus JR, Wilson-Sali T, Hsieh TS, 2005. Drosophila melanogaster topoisomerase III $\alpha$ preferentially relaxes a positively or negatively supercoiled bubble substrate and is essential during development. $J$ Biol Chem 280: 3564-3573.

Plank JL, Wu J, Hsieh TS. 2006. Topoisomerase III $\alpha$ and Bloom's helicase can resolve a mobile double Holliday junction substrate through convergent branch migration. Proc Natl Acad Sci 103: 11118-11123.

Raynard S, Bussen W, Sung P. 2006. A double Holliday junction dissolvasome comprising BLM, topoisomerase III $\alpha$, and BLAP75. J Biol Chem 281: 13861-13864.

Raynard S, Zhao W, Bussen W, Lu L, Ding YY, Busygina V, Meetei AR, Sung P. 2008. Functional role of BLAP75 in BLM-topoisomerase III $\alpha$-dependent Holliday junction processing. J Biol Chem 283: 15701-15708.

Seki M, Nakagawa T, Seki T, Kato G, Tada S, Takahashi Y, Yoshimura A, Kobayashi T, Aoki A, Otsuki M, et al. 2006. Bloom helicase and DNA topoisomerase III $\alpha$ are involved in the dissolution of sister chromatids. Mol Cell Biol 26: 6299-6307.

Shor E, Gangloff S, Wagner M, Weinstein J, Price G, Rothstein R. 2002. Mutations in homologous recombination genes rescue top3 slow growth in Saccharomyces cerevisiae. Genetics 162: 647-662.

Singh TR, Ali AM, Busygina V, Raynard S, Fan Q, Du CH, Andreassen PR, Sung P, Meetei AR. 2008. BLAP18/ RMI2, a novel OB-fold-containing protein, is an essential component of the Bloom helicase-double Holliday junction dissolvasome. Genes Dev 22: 2856-2868.

Srivenugopal KS, Lockshon D, Morris DR. 1984. Escherichia coli DNA topoisomerase III: Purification and characterization of a new type I enzyme. Biochemistry 23: 18991906.

Suski C, Marians KJ. 2008. Resolution of converging replication forks by RecQ and topoisomerase III. Mol Cell 30: 779-789.

Tong AH, Lesage G, Bader GD, Ding H, Xu H, Xin X, Young J, Berriz GF, Brost RL, Chang M, et al. 2004. Global mapping of the yeast genetic interaction network. Science 303: $808-813$.

Trowbridge K, McKim K, Brill SJ, Sekelsky J. 2007. Synthetic lethality of Drosophila in the absence of the MUS81 endonuclease and the DmBlm helicase is associated with elevated apoptosis. Genetics 176: 1993-2001.

Ui A, Satoh Y, Onoda F, Miyajima A, Seki M, Enomoto T. 2001. The N-terminal region of Sgs1, which interacts with Top3, is required for complementation of MMS sensitivity and suppression of hyper-recombination in sgs1 disruptants. Mol Genet Genomics 265: 837-850.

Viard T, de la Tour CB. 2007. Type IA topoisomerases: A simple puzzle? Biochimie 89: 456-467. 


\section{A.H. Bizard and I.D. Hickson}

Vindigni A, Hickson ID. 2009. RecQ helicases: Multiple structures for multiple functions? HFSP J 3: 153-164.

Wallis JW, Chrebet G, Brodsky G, Rolfe M, Rothstein R. 1989. A hyper-recombination mutation in S. cerevisiae identifies a novel eukaryotic topoisomerase. Cell 58: 409-419.

Wang JC. 2002. Cellular roles of DNA topoisomerases: A molecular perspective. Nat Rev Mol Cell Biol 3: 430-440.

Wang JC, Caron PR, Kim RA. 1990. The role of DNA topoisomerases in recombination and genome stability: A double-edged sword? Cell 62: 403-406.

Wang F, Yang Y, Singh TR, Busygina V, Guo R, Wan K, Wang W, Sung P, Meetei AR, Lei M. 2010. Crystal structures of RMI1 and RMI2, two OB-fold regulatory subunits of the BLM complex. Structure 18: 1159-1170.

Watt PM, Hickson ID, Borts RH, Louis EJ. 1996. SGS1, a homologue of the Bloom's and Werner's syndrome genes, is required for maintenance of genome stability in Saccharomyces cerevisiae. Genetics 144: 935-945.

Wechsler T, Newman S, West SC. 2011. Aberrant chromosome morphology in human cells defective for Holliday junction resolution. Nature 471: 642-646.

Weinstein J, Rothstein R. 2008. The genetic consequences of ablating helicase activity and the Top3 interaction domain of Sgs1. DNA Repair 7: 558-571.

Wu L, Hickson ID. 2001. RecQ helicases and topoisomerases: Components of a conserved complex for the regulation of genetic recombination. Cell Mol Life Sci 58: 894-901.

Wu L, Hickson ID. 2002. The Bloom's syndrome helicase stimulates the activity of human topoisomerase III $\alpha$. Nucleic Acids Res 30: 4823-4829.

Wu L, Hickson ID. 2003. The Bloom's syndrome helicase suppresses crossing over during homologous recombination. Nature 426: 870-874.

Wu L, Karow JK, Hickson ID. 1999. Genetic recombination: Helicases and topoisomerases link up. Curr Biol 9: R518520.

Wu L, Davies SL, North PS, Goulaouic H, Riou JF, Turley H, Gatter KC, Hickson ID. 2000. The Bloom's syndrome gene product interacts with topoisomerase III. J Biol Chem 275: 9636-9644.

Wu L, Chan KL, Ralf C, Bernstein DA, Garcia PL, Bohr VA, Vindigni A, Janscak P, Keck JL, Hickson ID. 2005. The HRDC domain of BLM is required for the dissolution of double Holliday junctions. EMBO J 24: 2679-2687.

Wu L, Bachrati CZ, Ou J, Xu C, Yin J, Chang M, Wang W, Li L, Brown GW, Hickson ID. 2006. BLAP75/RMI1 promotes the BLM-dependent dissolution of homologous recombination intermediates. Proc Natl Acad Sci 103: 4068-4073.

* Wyatt HDM, West SC. 2014. Holliday junction resolvases. Cold Spring Harb Perspect Biol doi: 10.1101/cshperspect .a016485.

Xu D, Guo R, Sobeck A, Bachrati CZ, Yang J, Enomoto T, Brown GW, Hoatlin ME, Hickson ID, Wang W. 2008. RMI, a new OB-fold complex essential for Bloom syndrome protein to maintain genome stability. Genes Dev 22: $2843-2855$.

Xue X, Raynard S, Busygina V, Singh AK, Sung P. 2013. Role of replication protein A in double Holliday junction dissolution mediated by the BLM-Topo III $\alpha$-RMI1-RMI2 protein complex. J Biol Chem 288: 14221-14227.

Yamagata K, Kato J, Shimamoto A, Goto M, Furuichi Y, Ikeda H. 1998. Bloom's and Werner's syndrome genes suppress hyperrecombination in yeast sgs 1 mutant: Implication for genomic instability in human diseases. Proc Natl Acad Sci 95: 8733-8738.

Yang J, Bachrati CZ, Ou J, Hickson ID, Brown GW. 2010. Human topoisomerase III $\alpha$ is a single-stranded DNA decatenase that is stimulated by BLM and RMI1. J Biol Chem 285: 21426-21436.

Yang J, Bachrati CZ, Hickson ID, Brown GW. 2012. BLM and RMI1 alleviate RPA inhibition of TopoIII $\alpha$ decatenase activity. PloS ONE 7: e41208.

Yin J, Sobeck A, Xu C, Meetei AR, Hoatlin M, Li L, Wang W. 2005. BLAP75, an essential component of Bloom's syndrome protein complexes that maintain genome integrity. EMBO J 24: 1465-1476. 


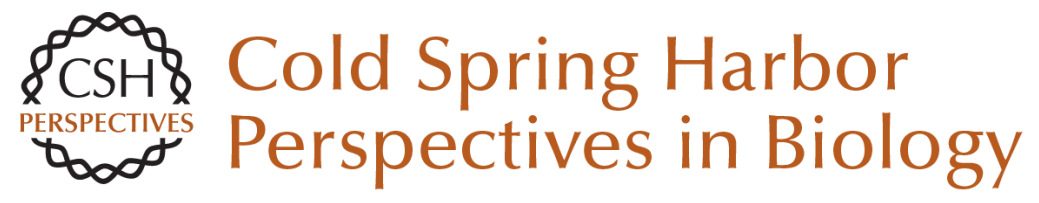

\section{The Dissolution of Double Holliday Junctions}

Anna H. Bizard and lan D. Hickson

Cold Spring Harb Perspect Biol 2014; doi: 10.1101/cshperspect.a016477

Subject Collection DNA Recombination

Meiotic Recombination: The Essence of Heredity Neil Hunter

Regulation of Recombination and Genomic Maintenance Wolf-Dietrich Heyer

Initiation of Meiotic Homologous Recombination: Flexibility, Impact of Histone Modifications, and Chromatin Remodeling Lóránt Székvölgyi, Kunihiro Ohta and Alain Nicolas

Mechanism and Regulation of Meiotic

Recombination Initiation Isabel Lam and Scott Keeney

Homologous Recombination and Human Health: The Roles of BRCA1, BRCA2, and Associated Proteins

Rohit Prakash, Yu Zhang, Weiran Feng, et al.

\section{Cell Biology of Mitotic Recombination}

Michael Lisby and Rodney Rothstein

DNA-Pairing and Annealing Processes in

Homologous Recombination and

Homology-Directed Repair

Scott W. Morrical

Mediators of Homologous DNA Pairing

Alex Zelensky, Roland Kanaar and Claire Wyman
An Overview of the Molecular Mechanisms of

Recombinational DNA Repair

Stephen C. Kowalczykowski

Recombination, Pairing, and Synapsis of Homologs during Meiosis

Denise Zickler and Nancy Kleckner

DNA Strand Exchange and RecA Homologs in Meiosis

M. Scott Brown and Douglas K. Bishop

Meiosis and Maternal Aging: Insights from Aneuploid Oocytes and Trisomy Births Mary Herbert, Dimitrios Kalleas, Daniel Cooney, et al.

Mismatch Repair during Homologous and Homeologous Recombination Maria Spies and Richard Fishel

Mechanisms of Gene Duplication and Amplification Andrew B. Reams and John R. Roth

The Role of Double-Strand Break Repair Pathways at Functional and Dysfunctional Telomeres Ylli Doksani and Titia de Lange

Regulation of DNA Pairing in Homologous Recombination James M. Daley, William A. Gaines, YoungHo Kwon, et al.

For additional articles in this collection, see http://cshperspectives.cshlp.org/cgi/collection/

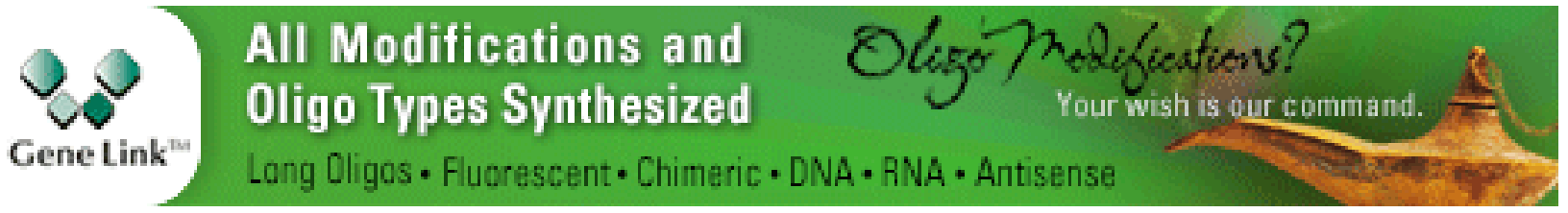


For additional articles in this collection, see http://cshperspectives.cshlp.org/cgi/collection/

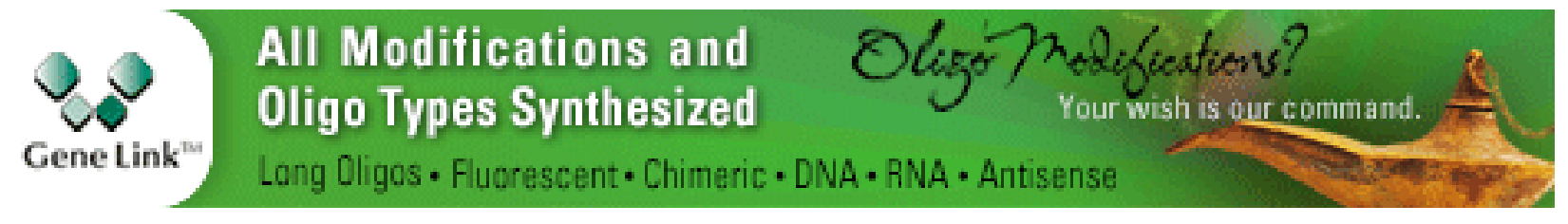

Copyright @ 2014 Cold Spring Harbor Laboratory Press; all rights reserved 